\title{
Uniformly asymptotic stability of almost periodic solutions for a delay difference system of plankton allelopathy
}

\section{Qinglong Wang and Zhijun Liu*}

"Correspondence:

zhijun_liu47@hotmail.com Department of Mathematics, Hubei University for Nationalities, Enshi, Hubei 445000, P.R. China

\begin{abstract}
In this contribution, we investigate a delayed difference almost periodic system for the growth of two species of plankton with competition and allelopathic effects on each other. By using the methods of Lyapunov function and preliminary lemmas, sufficient conditions which guarantee the existence and uniformly asymptotic stability of a unique positive almost periodic solution of the system are established. An example together with its numerical simulations is presented to verify the validity of the proposed criteria.
\end{abstract}

Keywords: delay difference system; allelopathy; almost periodic solutions; uniformly asymptotic stability; Lyapunov function

\section{Introduction}

Allelopathy is a biological phenomenon by which individuals of a population release one or more biochemicals that have an effect on the growth, survival, and reproduction of the individuals of another population. As an important factor for ecosystem functioning, allelopathic interactions have occurred in various aspects: between bacteria [1], between bacteria and phytoplankton [2,3], between phytoplankton and zooplankton [4], and also between calanoid copepods [5]. Especially, allelopathic interactions are widespread in phytoplankton communities, which deeply attract the attention of researchers. Thus, in aquatic ecology, the study of tremendous fluctuations in abundance of many phytoplankton communities is a significant theme. Recently, many workers have been aware that the increased population of one species of phytoplankton might restrain the growth of one or several other species by the production of allelopathic toxins. For detailed literature studies, we can refer to [6-15] and the references cited therein.

In [15], Qin and Liu discussed the permanence and global attractivity of the following delay difference system with plankton allelopathy:

$$
\left\{\begin{aligned}
& x_{1}(n+1)= x_{1}(n) \exp \left\{r_{1}(n)-a_{11}(n) x_{1}(n)-a_{12}(n) x_{2}(n)\right. \\
&\left.-b_{1}(n) x_{1}(n) \sum_{p=0}^{M} k_{2}(p) x_{2}(n-p)\right\}, \\
& x_{2}(n+1)= x_{2}(n) \exp \left\{r_{2}(n)-a_{21}(n) x_{1}(n)-a_{22}(n) x_{2}(n)\right. \\
&\left.-b_{2}(n) x_{2}(n) \sum_{p=0}^{M} k_{1}(p) x_{1}(n-p)\right\}, \\
& x_{i}(\Phi) \geq 0, \quad \Phi \in[-p, 0] \cap \mathbb{Z} ; \quad x_{i}(0)>0, \quad i=1,2,
\end{aligned}\right.
$$


where $x_{i}(n)$ are the population densities of species $x_{i}$ at the $n$th generation, $r_{i}(n)$ stand for the intrinsic growth rates of species $x_{i}$ at the $n$th generation, $a_{i i}(n)$ are the intra-specific effects of the $n$th generation of species $x_{i}$ on own population, and $a_{i j}(n)$ measure the interspecific effects of the $n$th generation of species $x_{j}$ on species $x_{i}, b_{i}(n) x_{i}(n) \sum_{p=0}^{M} k_{j}(p) x_{j}(n-$ $p$ ) denote the effect of toxic substances $(i, j=1,2 ; i \neq j), M$ is a positive integer.

Notice that the environment varies due to the factors such as seasonal effects and variations in weather conditions, food supplies, mating habits, harvesting etc. Thus it is reasonable to assume that the parameters in system (1.1) are periodic. However, if the various constituent components of the temporally nonuniform environment is with incommensurable periods (non-integral multiples), then we have to consider the environment to be almost periodic, which leads to the almost periodicity of the parameters of system (1.1). The main purpose is to establish sufficient conditions for the existence and uniformly asymptotic stability of a unique positive almost periodic solution of system (1.1). To do so, we assume that $\left\{r_{i}(n)\right\},\left\{a_{i j}(n)\right\}$ and $\left\{b_{i}(n)\right\}$ for $i, j=1,2$ are bounded nonnegative almost periodic sequences, $k_{i}(p), i=1,2$, is a bounded positive sequence.

Many recent works have been done on the existence and stability of almost periodic solutions for the discrete biological models without or with time delays (see [16-21]). However, to the best of our knowledge, there are few published papers concerning the above almost periodic system (1.1). For the sake of simplicity and convenience, in the following discussion, the notations below will be used

$$
h^{u}=\sup _{n \in \mathbb{Z}^{+}}\{h(n)\}, \quad h^{l}=\inf _{n \in \mathbb{Z}^{+}}\{h(n)\},
$$

where $\{h(n)\}$ is a bounded sequence defined on the set of nonnegative integers $\mathbb{Z}^{+}$. Meanwhile, we make a convention that $\sum_{n=a}^{b} h(n)=0$ if $a>b$.

The rest of this paper is organized as follows. In Section 2, we introduce some notations, definitions and lemmas which are useful for our main results. Sufficient conditions for the existence and uniformly asymptotic stability of a unique positive almost periodic solution of system (1.1) are established in Section 3. In Section 4, an example and its numerical simulations are presented to illustrate the feasibility of our main results. Finally, we give some proofs of theorems in the appendices for convenience in reading.

\section{Preliminaries}

In this section, we give some notations, definitions and lemmas which will be useful for the later sections.

Denote by $\mathbb{R}, \mathbb{R}^{+}, \mathbb{Z}$ and $\mathbb{Z}^{+}$the sets of real numbers, nonnegative real numbers, integers and nonnegative integers, respectively. $\mathbb{R}^{2}$ and $\mathbb{R}^{k}$ denote the cone of a two-dimensional and $k$-dimensional real Euclidean space, respectively. We also set

$$
[c, d]_{\mathbb{Z}}=[c, d] \cap \mathbb{Z}, \quad c, d \in \mathbb{Z}, \quad \mathbb{K}=[-M,+\infty)_{\mathbb{Z}}
$$

where $M$ is defined in (1.1).

Definition 2.1 (see [22]) A sequence $y: \mathbb{Z} \rightarrow \mathbb{R}^{k}$ is called an almost periodic sequence if the $\varepsilon$-translation set of $y$

$$
\mathcal{E}\{\varepsilon, y\}:=\{\tau \in \mathbb{Z}:|y(n+\tau)-y(n)|<\varepsilon, \forall n \in \mathbb{Z}\}
$$


is a relatively dense set in $\mathbb{Z}$ for all $\varepsilon>0$; that is, for any given $\varepsilon>0$, there exists an integer $l(\varepsilon)>0$ such that each interval of length $l(\varepsilon)$ contains an integer $\tau=\tau(\varepsilon) \in \mathcal{E}\{\varepsilon, y\}$ such that

$$
|y(n+\tau)-y(n)|<\varepsilon, \quad \forall n \in \mathbb{Z} .
$$

$\tau$ is called the $\varepsilon$-translation number of $y(n)$.

Definition 2.2 (see [22]) Let $g: \mathbb{Z} \times \mathbb{D} \rightarrow \mathbb{R}^{k}$, where $\mathbb{D}$ is an open set in $\mathbb{C}:=\{\phi$ : $\left.[-\tau, 0]_{\mathbb{Z}} \rightarrow \mathbb{R}^{k}\right\} . g(n, \phi)$ is said to be almost periodic in $n$ uniformly for $\phi \in \mathbb{D}$ if for any $\varepsilon>0$ and any compact set $\mathbb{S}$ in $\mathbb{D}$, there exists a positive integer $l(\varepsilon, \mathbb{S})$ such that any interval of length $l(\varepsilon, \mathbb{S})$ contains an integer $\tau$ for which

$$
|g(n+\tau, \phi)-g(n, \phi)|<\varepsilon, \quad \forall n \in \mathbb{Z}, \phi \in \mathbb{S} .
$$

$\tau$ is called the $\varepsilon$-translation number of $g(n, \phi)$.

Lemma 2.3 (see [23]) $\{y(n)\}$ is an almost periodic sequence if and only iffor any sequence $\left\{p_{k}^{\prime}\right\} \subset \mathbb{Z}$ there exists a subsequence $\left\{p_{k}\right\} \subset\left\{p_{k}^{\prime}\right\}$ such that $y\left(n+p_{k}\right)$ converges uniformly on $n \in \mathbb{Z}$ as $k \rightarrow \infty$. Furthermore, the limit sequence is also an almost periodic sequence.

Consider the following almost periodic delay difference system:

$$
y(n+1)=g\left(n, y_{n}\right), \quad n \in \mathbb{Z}^{+},
$$

where

$$
g: \mathbb{Z}^{+} \times \mathbb{C}_{B} \rightarrow \mathbb{R}, \quad \mathbb{C}_{B}=\{\phi \in \mathbb{C}:\|\phi\|<B\}, \mathbb{C}=\left\{\phi:[-\tau, 0]_{\mathbb{Z}} \rightarrow \mathbb{R}\right\},
$$

with $\|\phi\|=\sup _{s \in[-\tau, 0]_{\mathbb{Z}}}|\phi(s)|, g(n, \phi)$ is almost periodic in $n$ uniformly for $\phi \in \mathbb{C}_{B}$ and is continuous in $\phi$, while $y_{n} \in \mathbb{C}_{B}$ is defined as $y_{n}(s)=y(n+s)$ for all $s \in[-\tau, 0]_{\mathbb{Z}}$.

The product system of (2.1) is in the form of

$$
y(n+1)=g\left(n, y_{n}\right), \quad z(n+1)=g\left(n, z_{n}\right) .
$$

A discrete Lyapunov function of (2.2) is a function $V: \mathbb{Z}^{+} \times \mathbb{C}_{B} \times \mathbb{C}_{B} \rightarrow \mathbb{R}^{+}$which is continuous in its second and third variables. Define the difference of $V$ along the solution of system (2.2) by

$$
\Delta V_{(2.2)}(n, \phi, \psi)=V\left(n+1, y_{n+1}(n, \phi), z_{n+1}(n, \psi)\right)-V(n, \phi, \psi),
$$

where $(y(n, \phi), z(n, \psi))$ is a solution of system (2.2) through $(n,(\phi, \psi)), \phi, \psi \in \mathbb{C}_{B}$. And Zhang and Zheng [22] obtained the following lemma.

Lemma 2.4 (see [22]) Suppose that there exists a Lyapunov function $V(n, \phi, \psi)$ satisfying the following conditions: 
(1) $a(|\phi(0)-\psi(0)|) \leq V(n, \phi, \psi) \leq b(\|\phi-\psi\|)$, where $a, b \in \mathcal{P}$ with $\mathcal{P}=\{\alpha:[0, \infty) \rightarrow[0, \infty) \mid \alpha(0)=0$ and $\alpha(u)$ is continuous, increasing in $u\}$.

(2) $\left|V\left(n, \phi_{1}, \psi_{1}\right)-V\left(n, \phi_{2}, \psi_{2}\right)\right| \leq L\left(\left\|\phi_{1}-\phi_{2}\right\|+\left\|\psi_{1}-\psi_{2}\right\|\right)$, where $L>0$ is a constant.

(3) $\Delta V_{(2.2)}(n, \phi, \psi) \leq-\gamma V(n, \phi, \psi)$, where $0<\gamma<1$ is a constant.

Moreover, if there exists a solution $y(n)$ of system (2.1) such that $\left\|y_{n}\right\| \leq B^{*}<B$ for all $n \in \mathbb{Z}^{+}$, then there exists a unique uniformly asymptotically stable almost periodic solution $q(n)$ of system (2.1) which satisfies $|q(n)| \leq B^{*}$ for all $n \in \mathbb{K}$. In particular, if $g(n, \phi)$ is periodic with period $\omega$, then system (2.1) has a unique uniformly asymptotically stable periodic solution with period $\omega$.

Remark 2.5 (see [19]) From the proof of [24, Theorem 6.6], it is not difficult to prove that condition (3) of Lemma 2.4 can be replaced by the following condition:

$(3)^{\prime} \Delta V_{(2.2)}(n, \phi, \psi) \leq-c(|\phi(0)-\psi(0)|)$, where $c \in\{\beta:[0, \infty) \rightarrow[0, \infty) \mid \beta$ is continuous, $\beta(0)=0$ and $\beta(s)>0$ for $s>0\}$.

Definition 2.6 (see [15]) System (1.1) is said to be permanent if there exist positive constants $\mathfrak{M}_{i}$ and $\mathcal{M}_{i}$ such that

$$
\mathfrak{M}_{i} \leq \liminf _{n \rightarrow+\infty} x_{i}(n) \leq \limsup _{n \rightarrow+\infty} x_{i}(n) \leq \mathcal{M}_{i}, \quad i=1,2
$$

for any positive solution $\left(x_{1}(n), x_{2}(n)\right)$ of system $(1.1)$.

Lemma 2.7 (see [15]) Assume that

$$
\begin{aligned}
& \min \left\{r_{1}^{l}-a_{12}^{u} M_{2}, r_{2}^{l}-a_{21}^{u} M_{1}\right\}>0, \\
& \min \left\{\Delta_{1} M_{1}, \Delta_{2} M_{2}\right\}>1
\end{aligned}
$$

Then system (1.1) is permanent. Here, $\Delta_{i}=\frac{a_{i i}^{u}+b_{i}^{u} M_{j}(M+1) k_{j}^{u}}{r_{i}^{l}-a_{i j}^{u} M_{j}}$.

From the proof of [15, Lemma 2.3], we have

$$
\limsup _{n \rightarrow+\infty} x_{i}(n) \leq M_{i} \stackrel{\text { def }}{=} \frac{\exp \left(r_{i}^{u}-1\right)}{a_{i i}^{l}}
$$

and

$$
\liminf _{n \rightarrow+\infty} x_{i}(n) \geq m_{i} \stackrel{\operatorname{def}}{=} \frac{\exp \left[\left(r_{i}^{l}-a_{i j}^{u} M_{j}\right)\left(1-\Delta_{i} M_{i}\right)\right]}{\Delta_{i}},
$$

where $i, j=1,2, i \neq j$.

\section{Main result}

According to (2.4) and (2.5), we denote by $\Omega$ the set of all solutions $\left(x_{1}(n), x_{2}(n)\right)$ of system (1.1) satisfying $m_{i} \leq x_{i}(n) \leq M_{i}, i=1,2$, for all $n \in \mathbb{K}$. From Lemma 2.4, we first prove that there exists a bounded solution of system (1.1) and then construct a suitable Lyapunov function for system (1.1). 
Theorem 3.1 If conditions (2.3) are satisfied, then $\Omega \neq \phi$.

The proof of Theorem 3.1 is given in Appendix 1.

Theorem 3.2 If conditions (2.3) and

$$
\begin{aligned}
& 1-\max \left(\left|1-a_{11}^{l} m_{1}\right|,\left|1-a_{11}^{u} M_{1}\right|\right)-\left(b_{1}^{u} k_{2}^{u}+b_{2}^{u} k_{1}^{u}\right)(M+1) M_{1} M_{2}-a_{21}^{u} M_{1}>0, \\
& 1-\max \left(\left|1-a_{22}^{l} m_{2}\right|,\left|1-a_{22}^{u} M_{2}\right|\right)-\left(b_{1}^{u} k_{2}^{u}+b_{2}^{u} k_{1}^{u}\right)(M+1) M_{1} M_{2}-a_{12}^{u} M_{2}>0
\end{aligned}
$$

are satisfied, then system (1.1) possesses a unique almost periodic solution $\left(x_{1}^{*}(n), x_{2}^{*}(n)\right)$, and it is uniformly asymptotically stable within $\Omega$.

The proof of Theorem 3.2 is given in Appendix 2 .

\section{Example and numerical simulations}

In this section, to verify the validity of our main results, we give an example and its corresponding numerical simulations.

Example 4.1 Consider the following discrete system with a delay:

$$
\left\{\begin{aligned}
x_{1}(n+1)= & x_{1}(n) \exp \left\{0.85+0.02 \sin (\sqrt{2} n \pi)-(0.80-0.01 \sin (\sqrt{2} n \pi)) x_{1}(n)\right. \\
& -(0.03+0.01 \sin (\sqrt{2} n \pi)) x_{2}(n) \\
& \left.-(0.02-0.01 \cos (\sqrt{2} n \pi)) x_{1}(n)\left[0.83 x_{2}(n)+0.83 x_{2}(n-1)\right]\right\} \\
x_{2}(n+1)= & x_{2}(n) \exp \{0.80+0.01 \cos (\sqrt{2} n \pi) \\
& -(0.02+0.01 \cos (\sqrt{2} n \pi)) x_{1}(n) \\
& -(0.65+0.02 \sin (\sqrt{2} n \pi)) x_{2}(n) \\
& \left.-(0.03+0.02 \sin (\sqrt{2} n \pi)) x_{2}(n)\left[0.73 x_{1}(n)+0.73 x_{1}(n-1)\right]\right\}
\end{aligned}\right.
$$

with the following initial conditions:

$$
x_{1}^{*}(-1)=1.06, \quad x_{1}^{*}(0)=1.02, \quad x_{2}^{*}(-1)=0.85, \quad x_{2}^{*}(0)=0.98 \text {. }
$$

By a computation, we get

$$
\begin{aligned}
& M_{1} \approx 1.1115, \quad M_{2} \approx 1.3126, \quad m_{1} \approx 0.7305, \\
& m_{2} \approx 0.8010, \quad \Delta_{1} \approx 1.1259, \quad \Delta_{2} \approx 0.9927, \\
& r_{1}^{l}-a_{12}^{u} M_{2} \approx 0.7775>0, \quad r_{2}^{l}-a_{21}^{u} M_{1} \approx 0.7567>0, \\
& \Delta_{1} M_{1} \approx 1.2514>1, \quad \Delta_{2} M_{2} \approx 1.3030>1
\end{aligned}
$$

and

$$
\min \left\{r_{1}^{l}-a_{12}^{u} M_{2}, r_{2}^{l}-a_{21}^{u} M_{1}\right\}>0, \quad \min \left\{\Delta_{1} M_{1}, \Delta_{2} M_{2}\right\}>1 .
$$



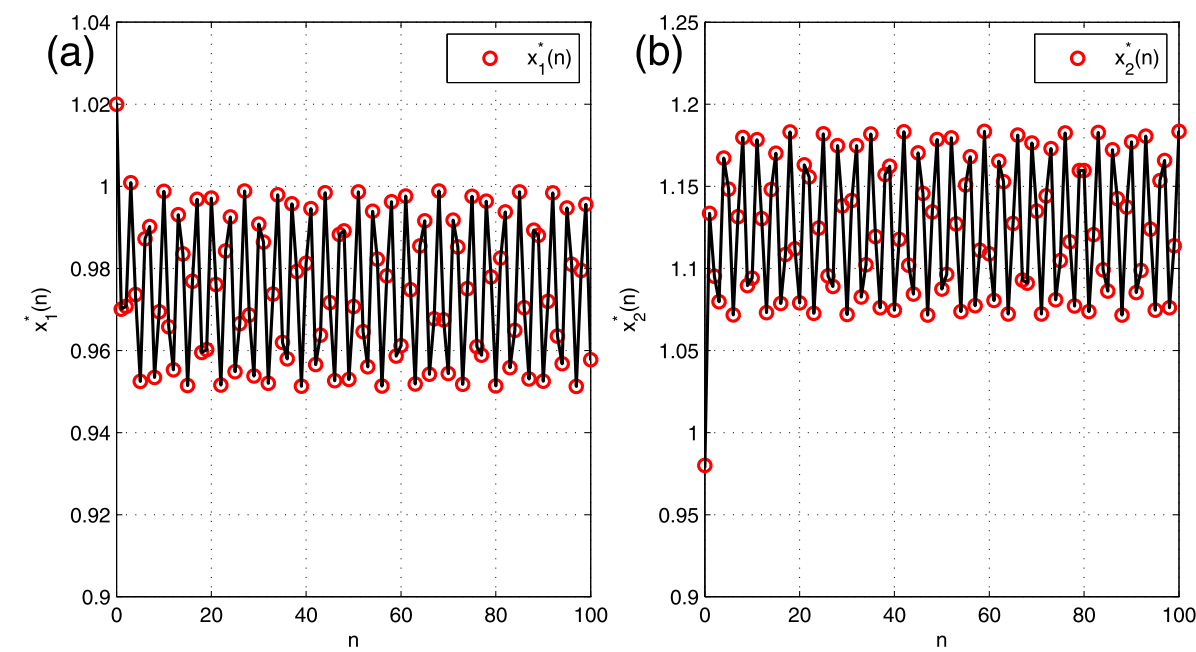

Figure 1 Positive almost periodic solution of system (4.1). (a) Time-series $x_{1}^{*}(n)$ with initial values $x_{1}^{*}(-1)=1.06, x_{1}^{*}(0)=1.02$ for $n \in[0,100]$. (b) Time-series $x_{2}^{*}(n)$ with initial values $x_{2}^{*}(-1)=0.85, x_{2}^{*}(0)=0.98$ for $n \in[0,100]$

A further calculation shows that

$$
\begin{aligned}
1 & -\max \left(\left|1-a_{11}^{l} m_{1}\right|,\left|1-a_{11}^{u} M_{1}\right|\right) \\
& \quad-\left(b_{1}^{u} k_{2}^{u}+b_{2}^{u} k_{1}^{u}\right)(M+1) M_{1} M_{2}-a_{21}^{u} M_{1} \approx 0.3646>0, \\
1 & -\max \left(\left|1-a_{22}^{l} m_{2}\right|,\left|1-a_{22}^{u} M_{2}\right|\right) \\
& \quad-\left(b_{1}^{u} k_{2}^{u}+b_{2}^{u} k_{1}^{u}\right)(M+1) M_{1} M_{2}-a_{12}^{u} M_{2} \approx 0.2729>0 .
\end{aligned}
$$

Clearly, the assumptions of Theorem 3.2 are satisfied, and hence system (4.1) has a unique uniformly asymptotically stable positive almost periodic solution. From Figure 1, we can see that there exists a positive almost periodic solution $\left(x_{1}^{*}(t), x_{2}^{*}(t)\right)$, and the twodimensional and three-dimensional phase portraits of almost periodic system (4.1) are revealed in Figure 2, respectively. Figure 3 shows that any positive solution $\left(x_{1}(n), x_{2}(n)\right)$ tends to the almost periodic solution $\left(x_{1}^{*}(n), x_{2}^{*}(n)\right)$.

\section{Appendix 1: Proof of Theorem 3.1}

By the almost periodicity of $\left\{r_{i}(n)\right\},\left\{a_{i j}(n)\right\}$ and $\left\{b_{i}(n)\right\}, i, j=1,2$, any sequence $\left\{\tau_{k}\right\} \subset \mathbb{Z}^{+}$, with $\tau_{k} \rightarrow+\infty$ as $k \rightarrow+\infty$, is such that

$$
r_{i}\left(n+\tau_{k}\right) \rightarrow r_{i}(n), \quad a_{i j}\left(n+\tau_{k}\right) \rightarrow a_{i j}(n), \quad b_{i}\left(n+\tau_{k}\right) \rightarrow b_{i}(n), \quad i, j=1,2,
$$

as $k \rightarrow+\infty$ for $n \in \mathbb{Z}^{+}$. Let $\varepsilon$ be an arbitrary small positive number. It follows from (2.4) and (2.5) that there exists a positive integer $N_{0}$ such that

$$
m_{i}-\varepsilon \leq x_{i}(n) \leq M_{i}+\varepsilon \quad \text { for all } n>N_{0} .
$$

Let $x_{i k}(n)=x_{i}\left(n+\tau_{k}\right)$ for $n \geq N_{0}+M-\tau_{k}, k=1,2, \ldots$. For any positive integer $q$, we can see that there exists a sequence $\left\{x_{i k}(n): k \geq q\right\}$ such that the sequence $\left\{x_{i k}(n)\right\}$ has a 

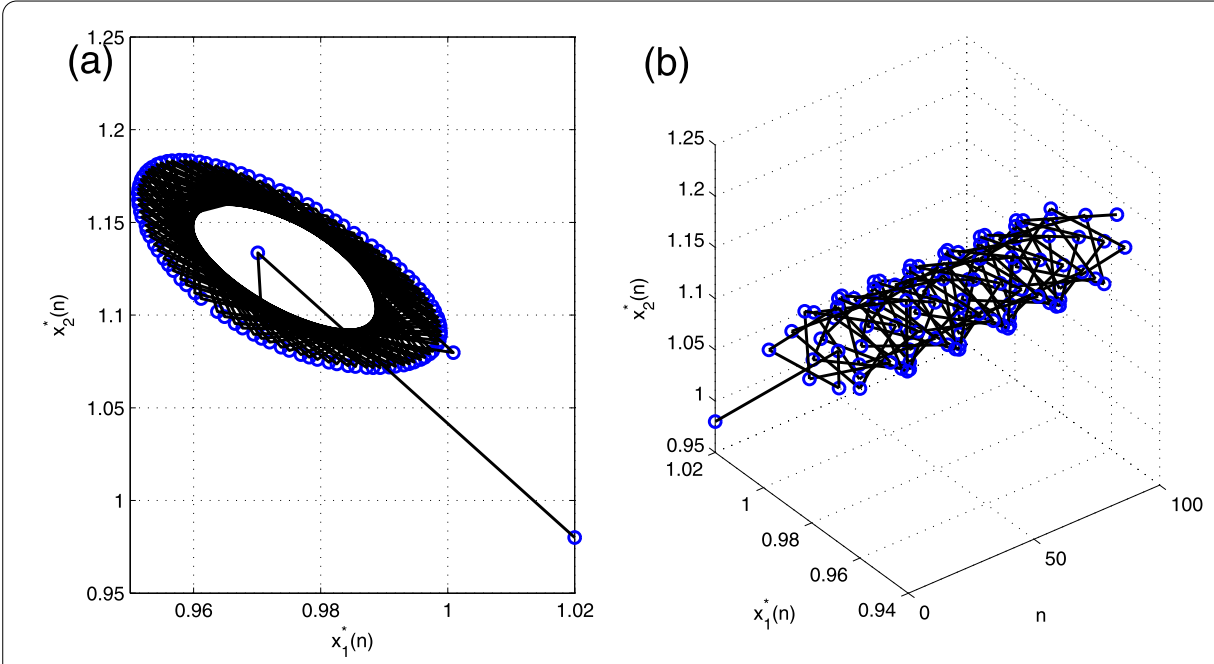

Figure 2 Phase portrait. (a) Two-dimensional phase portrait of almost periodic system (4.1). Time-series $x_{1}^{*}(n)$ and $x_{2}^{*}(n)$ with initial values $x_{1}^{*}(-1)=1.06, x_{1}^{*}(0)=1.02, x_{2}^{*}(-1)=0.85, x_{2}^{*}(0)=0.98$ for $n \in[0,100]$. (b) Three-dimensional phase portrait of almost periodic system (4.1). Time-series $x_{1}^{*}(n)$ and $x_{2}^{*}(n)$ with the above initial values for $n \in[0,100]$.

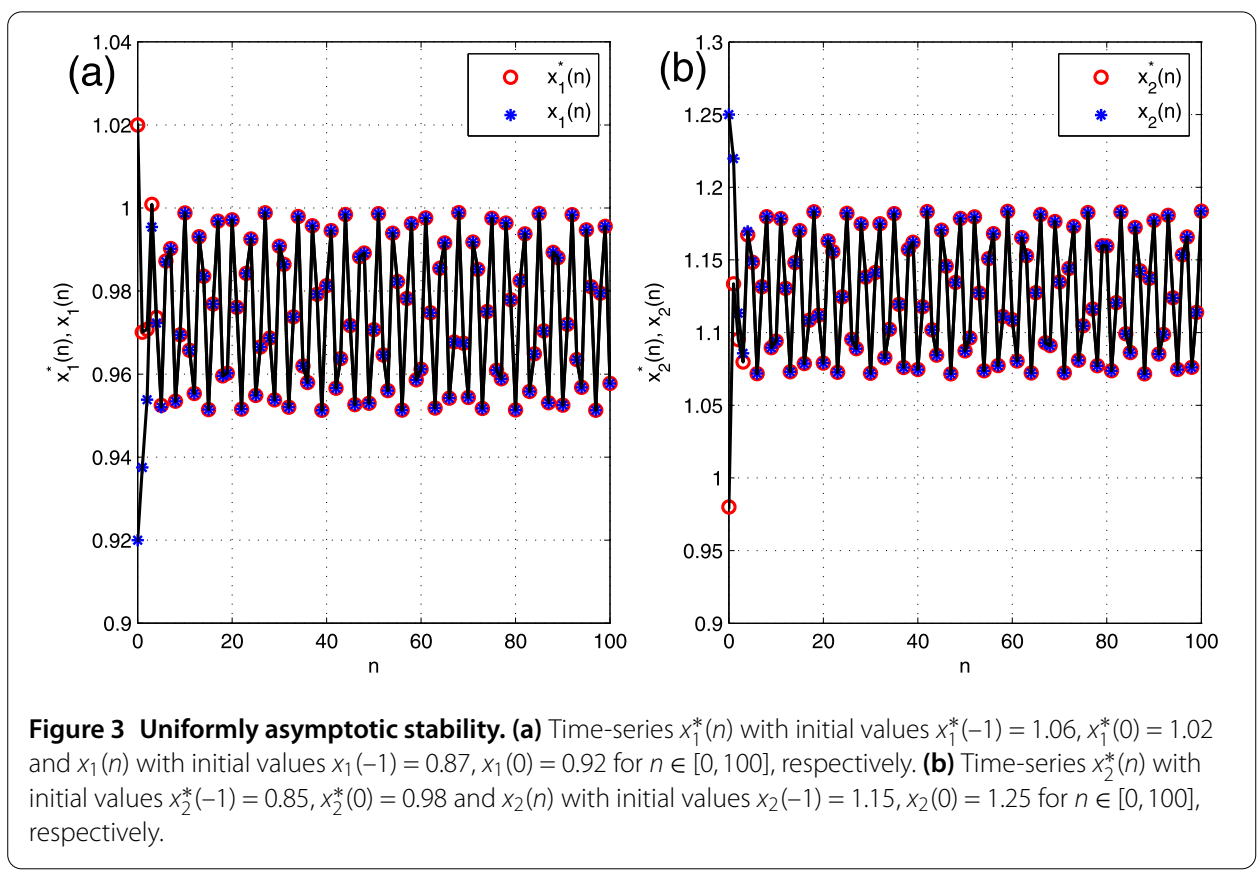


subsequence, denoted by $\left\{x_{i k}(n)\right\}$ again, converging on any finite interval of $\mathbb{K}$ as $k \rightarrow+\infty$. So, we have a sequence $\left\{y_{i}(n)\right\}, i=1,2$, satisfying

$$
x_{i k}(n) \rightarrow y_{i}(n) \quad \text { for } n \in \mathbb{K} \text { as } k \rightarrow+\infty,
$$

which, together with (A.1) and

$$
\left\{\begin{aligned}
x_{1 k}(n+1)= & x_{1 k}(n) \exp \left\{r_{1}\left(n+\tau_{k}\right)-a_{11}\left(n+\tau_{k}\right) x_{1 k}(n)-a_{12}\left(n+\tau_{k}\right) x_{2 k}(n)\right. \\
& \left.-b_{1}\left(n+\tau_{k}\right) x_{1 k}(n) \sum_{p=0}^{M} k_{2}(p) x_{2 k}(n-p)\right\}, \\
x_{2 k}(n+1)= & x_{2 k}(n) \exp \left\{r_{2}\left(n+\tau_{k}\right)-a_{21}\left(n+\tau_{k}\right) x_{1 k}(n)-a_{22}\left(n+\tau_{k}\right) x_{2 k}(n)\right. \\
& \left.-b_{2}\left(n+\tau_{k}\right) x_{2 k}(n) \sum_{p=0}^{M} k_{1}(p) x_{1 k}(n-p)\right\},
\end{aligned}\right.
$$

yields

$$
\left\{\begin{aligned}
y_{1}(n+1)= & y_{1}(n) \exp \left\{r_{1}(n)-a_{11}(n) y_{1}(n)-a_{12}(n) y_{2}(n)\right. \\
& \left.-b_{1}(n) y_{1}(n) \sum_{p=0}^{M} k_{2}(p) y_{2}(n-p)\right\} \\
y_{2}(n+1)= & y_{2}(n) \exp \left\{r_{2}(n)-a_{21}(n) y_{1}(n)-a_{22}(n) y_{2}(n)\right. \\
& \left.-b_{2}(n) y_{2}(n) \sum_{p=0}^{M} k_{1}(p) y_{1}(n-p)\right\} .
\end{aligned}\right.
$$

We can easily see that $\left(y_{1}(n), y_{2}(n)\right)$ is a solution of system (1.1) and $m_{i}-\varepsilon \leq y_{i}(n) \leq M_{i}+\varepsilon$ for $n \in \mathbb{K}$. Since $\varepsilon$ is small enough, it follows that

$$
m_{i} \leq y_{i}(n) \leq M_{i}, \quad i=1,2 \text { for } n \in \mathbb{K} .
$$

This completes the proof.

\section{Appendix 2: Proof of Theorem 3.2}

We first make the change of variables

$$
p_{1}(n)=\ln x_{1}(n), \quad p_{2}(n)=\ln x_{2}(n)
$$

It follows from system (1.1) that

$$
\left\{\begin{aligned}
p_{1}(n+1)= & p_{1}(n)+r_{1}(n)-a_{11}(n) e^{p_{1}(n)}-a_{12}(n) e^{p_{2}(n)} \\
& -b_{1}(n) e^{p_{1}(n)} \sum_{p=0}^{M} k_{2}(p) e^{p_{2}(n-p)} \\
p_{2}(n+1)= & p_{2}(n)+r_{2}(n)-a_{21}(n) e^{p_{1}(n)}-a_{22}(n) e^{p_{2}(n)} \\
& -b_{2}(n) e^{p_{2}(n)} \sum_{p=0}^{M} k_{1}(p) e^{p_{1}(n-p)}
\end{aligned}\right.
$$

From Theorem 3.1, it is easy to see that system (B.1) has a bounded solution $\left(p_{1}(n), p_{2}(n)\right)$ satisfying

$$
\ln m_{1} \leq p_{1}(n) \leq \ln M_{1}, \quad \ln m_{2} \leq p_{2}(n) \leq \ln M_{2} \quad \text { for all } n \in \mathbb{K}
$$

Thus $\left|p_{1}(n)\right| \leq A_{1},\left|p_{2}(n)\right| \leq A_{2}$, where $A_{i}=\max \left\{\left|\ln m_{i}\right|,\left|\ln M_{i}\right|\right\}, i=1,2$. Suppose that $Y_{n}(s)=\left(p_{1}(n+s), p_{2}(n+s)\right), Z_{n}(s)=\left(q_{1}(n+s), q_{2}(n+s)\right)\left(n \in \mathbb{Z}^{+}, s \in[-M, 0]_{\mathbb{Z}}\right)$ are any two 
solutions of system (B.1) defined on $\mathbb{S}$, where $\mathbb{S}=\left\{\left(p_{1}(n), p_{2}(n)\right) \mid \ln m_{i} \leq p_{i}(n) \leq \ln M_{i}, i=\right.$ $1,2, n \in \mathbb{K}\}$. Define the norm

$$
\left\|Y_{n}(s)\right\|=\left\|\left(p_{1}(n+s), p_{2}(n+s)\right)\right\|=\sup _{s \in[-M, 0]_{\mathbb{Z}}}\left\{\left|p_{1}(n+s)\right|+\left|p_{2}(n+s)\right|\right\},
$$

where $\left(p_{1}(n+s), p_{2}(n+s)\right) \in \mathbb{R}^{2}$, then

$$
\left\|Y_{n}\right\| \leq B, \quad\left\|Z_{n}\right\| \leq B,
$$

where $B=A_{1}+A_{2}$. Consider the associate product system of system (B.1)

$$
\left\{\begin{aligned}
p_{1}(n+1)= & p_{1}(n)+r_{1}(n)-a_{11}(n) e^{p_{1}(n)}-a_{12}(n) e^{p_{2}(n)} \\
& -b_{1}(n) e^{p_{1}(n)} \sum_{p=0}^{M} k_{2}(p) e^{p_{2}(n-p)}, \\
p_{2}(n+1)= & p_{2}(n)+r_{2}(n)-a_{21}(n) e^{p_{1}(n)}-a_{22}(n) e^{p_{2}(n)} \\
& -b_{2}(n) e^{p_{2}(n)} \sum_{p=0}^{M} k_{1}(p) e^{p_{1}(n-p)}, \\
q_{1}(n+1)= & q_{1}(n)+r_{1}(n)-a_{11}(n) e^{q_{1}(n)}-a_{12}(n) e^{q_{2}(n)} \\
& -b_{1}(n) e^{q_{1}(n)} \sum_{p=0}^{M} k_{2}(p) e^{q_{2}(n-p)}, \\
q_{2}(n+1)= & q_{2}(n)+r_{2}(n)-a_{21}(n) e^{q_{1}(n)}-a_{22}(n) e^{q_{2}(n)} \\
& -b_{2}(n) e^{q_{2}(n)} \sum_{p=0}^{M} k_{1}(p) e^{q_{1}(n-p)} .
\end{aligned}\right.
$$

Construct a Lyapunov function $V(n)=V\left(n, Y_{n}, Z_{n}\right)$ defined on $\mathbb{Z}^{+} \times \mathbb{S} \times \mathbb{S}$ as follows:

$$
\begin{aligned}
V(n)= & V\left(n, Y_{n}, Z_{n}\right) \\
= & \left|p_{1}(n)-q_{1}(n)\right|+\left|p_{2}(n)-q_{2}(n)\right| \\
& +\sum_{p=0}^{M} \sum_{m=n-p}^{n-1} b_{2}^{u} k_{1}^{u} M_{1} M_{2}\left|p_{1}(m)-q_{1}(m)\right| \\
& +\sum_{p=0}^{M} \sum_{m=n-p}^{n-1} b_{1}^{u} k_{2}^{u} M_{1} M_{2}\left|p_{2}(m)-q_{2}(m)\right| .
\end{aligned}
$$

It is easy to see that

$$
\begin{aligned}
\left|Y_{n}(0)-Z_{n}(0)\right| \leq & V(n) \\
\leq & \left|p_{1}(n)-q_{1}(n)\right|+\left|p_{2}(n)-q_{2}(n)\right| \\
& +\sum_{p=0}^{M} \sum_{m=n-p}^{n-1} D\left\{\left|p_{1}(m)-q_{1}(m)\right|+\left|p_{2}(m)-q_{2}(m)\right|\right\} \\
\leq & {\left[1+\frac{(1+M) M D}{2}\right] \sup _{s \in[-M, 0]]_{\mathbb{Z}}}\left\{\left|p_{1}(n+s)-q_{1}(n+s)\right|\right.} \\
& \left.+\left|p_{2}(n+s)-q_{2}(n+s)\right|\right\} \\
= & \lambda\left\|Y_{n}-Z_{n}\right\|,
\end{aligned}
$$


where

$$
\begin{aligned}
\left|Y_{n}(0)-Z_{n}(0)\right| & =\sqrt{\left(p_{1}(n)-q_{1}(n)\right)^{2}+\left(p_{2}(n)-q_{2}(n)\right)^{2}} \\
& \leq\left|p_{1}(n)-q_{1}(n)\right|+\left|p_{2}(n)-q_{2}(n)\right|,
\end{aligned}
$$

and $\lambda=1+\frac{(1+M) M D}{2}, D=\max \left\{b_{1}^{u} k_{2}^{u} M_{1} M_{2}, b_{2}^{u} k_{1}^{u} M_{1} M_{2}\right\}$. Let $a, b \in C\left(\mathbb{R}^{+}, \mathbb{R}^{+}\right), a(x)=x, b(x)=$ $\lambda x$, so condition (1) in Lemma 2.4 is satisfied.

For $\forall y, z, \tilde{y}, \tilde{z} \in \mathbb{R}$, one has

$$
\begin{aligned}
|| y-z|-| \tilde{y}-\tilde{z}|| & = \begin{cases}|y-z|-|\tilde{y}-\tilde{z}|, & \text { if }|y-z| \geq|\tilde{y}-\tilde{z}|, \\
|\tilde{y}-\tilde{z}|-|y-z|, & \text { if }|\tilde{y}-\tilde{z}|>|y-z|\end{cases} \\
& \leq \begin{cases}|(y-\tilde{y})+(\tilde{z}-z)|, & \text { if }|y-z| \geq|\tilde{y}-\tilde{z}|, \\
|(\tilde{y}-y)+(z-\tilde{z})|, & \text { if }|\tilde{y}-\tilde{z}|>|y-z|\end{cases} \\
& \leq \begin{cases}|y-\tilde{y}|+|\tilde{z}-z|, & \text { if }|y-z| \geq|\tilde{y}-\tilde{z}|, \\
|\tilde{y}-y|+|z-\tilde{z}|, & \text { if }|\tilde{y}-\tilde{z}|>|y-z|\end{cases} \\
& =|y-\tilde{y}|+|z-\tilde{z}| .
\end{aligned}
$$

Hence, for $\forall Y_{n}, Z_{n}, \tilde{Y}_{n}, \tilde{Z}_{n} \in \mathbb{S}$, by (B.7) we have

$$
\begin{aligned}
\mid V(n, & \left.Y_{n}, Z_{n}\right)-V\left(n, \tilde{Y}_{n}, \tilde{Z}_{n}\right) \mid \\
= & || p_{1}(n)-q_{1}(n)|+| p_{2}(n)-q_{2}(n)\left|+\sum_{p=0}^{M} \sum_{m=n-p}^{n-1} b_{2}^{u} k_{1}^{u} M_{1} M_{2}\right| p_{1}(m)-q_{1}(m) \mid \\
& +\sum_{p=0}^{M} \sum_{m=n-p}^{n-1} b_{1}^{u} k_{2}^{u} M_{1} M_{2}\left|p_{2}(m)-q_{2}(m)\right| \\
& -\left|\tilde{p}_{1}(n)-\tilde{q}_{1}(n)\right|-\left|\tilde{p}_{2}(n)-\tilde{q}_{2}(n)\right|-\sum_{p=0}^{M} \sum_{m=n-p}^{n-1} b_{2}^{u} k_{1}^{u} M_{1} M_{2}\left|\tilde{p}_{1}(m)-\tilde{q}_{1}(m)\right| \\
& -\sum_{p=0}^{M} \sum_{m=n-p}^{n-1} b_{1}^{u} k_{2}^{u} M_{1} M_{2}\left|\tilde{p}_{2}(m)-\tilde{q}_{2}(m)\right| \mid \\
\leq & || p_{1}(n)-q_{1}(n)|+| p_{2}(n)-q_{2}(n)|-| \tilde{p}_{1}(n)-\tilde{q}_{1}(n)|-| \tilde{p}_{2}(n)-\tilde{q}_{2}(n)|| \\
& +\sum_{p=0}^{M} \sum_{m=n-p}^{n-1} b_{2}^{u} k_{1}^{u} M_{1} M_{2}|| p_{1}(m)-q_{1}(m)|-| \tilde{p}_{1}(m)-\tilde{q}_{1}(m)|| \\
& +\sum_{p=0}^{M} \sum_{m=n-p}^{n-1} b_{1}^{u} k_{2}^{u} M_{1} M_{2}|| p_{2}(m)-q_{2}(m)|-| \tilde{p}_{2}(m)-\tilde{q}_{2}(m)|| \\
\leq & \left\{\left|p_{1}(n)-\tilde{p}_{1}(n)\right|+\left|p_{2}(n)-\tilde{p}_{2}(n)\right|+\left|q_{1}(n)-\tilde{q}_{1}(n)\right|+\left|q_{2}(n)-\tilde{q}_{2}(n)\right|\right\} \\
& +\sum_{p=0}^{M} \sum_{m=n-p}^{n-1} b_{2}^{u} k_{1}^{u} M_{1} M_{2}\left\{\left|p_{1}(m)-\tilde{p}_{1}(m)\right|+\left|q_{1}(m)-\tilde{q}_{1}(m)\right|\right\}
\end{aligned}
$$




$$
\begin{aligned}
& +\sum_{p=0}^{M} \sum_{m=n-p}^{n-1} b_{1}^{u} k_{2}^{u} M_{1} M_{2}\left\{\left|p_{2}(m)-\tilde{p}_{2}(m)\right|+\left|q_{2}(m)-\tilde{q}_{2}(m)\right|\right\} \\
\leq & {\left[1+\frac{(1+M) M D}{2}\right] \sup _{s \in[-M, 0]_{\mathbb{Z}}}\left\{\left|p_{1}(n+s)-\tilde{p}_{1}(n+s)\right|+\left|p_{2}(n+s)-\tilde{p}_{2}(n+s)\right|\right.} \\
& \left.+\left|q_{1}(n+s)-\tilde{q}_{1}(n+s)\right|+\left|q_{2}(n+s)-\tilde{q}_{2}(n+s)\right|\right\} \\
\leq & \lambda\left(\left\|Y_{n}-\tilde{Y}_{n}\right\|+\left\|Z_{n}-\tilde{Z}_{n}\right\|\right),
\end{aligned}
$$

where $\lambda=1+\frac{(1+M) M D}{2}, D=\max \left\{b_{1}^{u} k_{2}^{u} M_{1} M_{2}, b_{2}^{u} k_{1}^{u} M_{1} M_{2}\right\}$. Condition (2) in Lemma 2.4 is also satisfied.

Using the mean-value theorem, we derive that

$$
\begin{aligned}
& e^{p_{i}(n)}-e^{q_{i}(n)}=\xi_{i}(n)\left(p_{i}(n)-q_{i}(n)\right), \\
& e^{p_{i}(n-p)}-e^{q_{i}(n-p)}=\eta_{i}(n-p)\left(p_{i}(n-p)-q_{i}(n-p)\right),
\end{aligned}
$$

$i=1,2$, where $\xi_{i}(n)$ lies between $e^{p_{i}(n)}$ and $e^{q_{i}(n)}$ and $\eta_{i}(n-p)$ lies between $e^{p_{i}(n-p)}$ and $e^{q_{i}(n-p)}$, respectively. So, $m_{i} \leq \xi_{i}(n), \eta_{i}(n-p) \leq M_{i}, n \in \mathbb{Z}^{+}$.

In view of system (B.3) together with (B.9) and (B.10), we have

$$
\begin{aligned}
\left|p_{1}(n+1)-q_{1}(n+1)\right|+\left|p_{2}(n+1)-q_{2}(n+1)\right| & \mid p_{1}(n)-q_{1}(n)-a_{11}(n)\left(e^{p_{1}(n)}-e^{q_{1}(n)}\right)-a_{12}(n)\left(e^{p_{2}(n)}-e^{q_{2}(n)}\right) \\
& -b_{1}(n)\left(e^{p_{1}(n)} \sum_{p=0}^{M} k_{2}(p) e^{p_{2}(n-p)}-e^{q_{1}(n)} \sum_{p=0}^{M} k_{2}(p) e^{q_{2}(n-p)}\right) \mid \\
& +\mid p_{2}(n)-q_{2}(n)-a_{21}(n)\left(e^{p_{1}(n)}-e^{q_{1}(n)}\right)-a_{22}(n)\left(e^{p_{2}(n)}-e^{q_{2}(n)}\right) \\
& -b_{2}(n)\left(e^{p_{2}(n)} \sum_{p=0}^{M} k_{1}(p) e^{p_{1}(n-p)}-e^{q_{2}(n)} \sum_{p=0}^{M} k_{1}(p) e^{q_{1}(n-p)}\right) \mid \\
= & \mid p_{1}(n)-q_{1}(n)-a_{11}(n)\left(e^{p_{1}(n)}-e^{q_{1}(n)}\right)-a_{12}(n)\left(e^{p_{2}(n)}-e^{q_{2}(n)}\right) \\
& -b_{1}(n)\left(e^{p_{1}(n)} \sum_{p=0}^{M} k_{2}(p) e^{p_{2}(n-p)}-e^{p_{1}(n)} \sum_{p=0}^{M} k_{2}(p) e^{q_{2}(n-p)}\right. \\
& \left.+e^{p_{1}(n)} \sum_{p=0}^{M} k_{2}(p) e^{q_{2}(n-p)}-e^{q_{1}(n)} \sum_{p=0}^{M} k_{2}(p) e^{q_{2}(n-p)}\right) \mid \\
& +\mid p_{2}(n)-q_{2}(n)-a_{21}(n)\left(e^{p_{1}(n)}-e^{q_{1}(n)}\right)-a_{22}(n)\left(e^{p_{2}(n)}-e^{q_{2}(n)}\right) \\
& \mid b_{p=0}^{M} e^{p_{2}(n)} \sum_{p=0}^{M} k_{1}(p) e^{p_{1}(n-p)}-e^{p_{2}(n)} \sum_{p}^{M}(p) e^{q_{1}(n-p)}
\end{aligned}
$$




$$
\begin{aligned}
& \left.+e^{p_{2}(n)} \sum_{p=0}^{M} k_{1}(p) e^{q_{1}(n-p)}-e^{q_{2}(n)} \sum_{p=0}^{M} k_{1}(p) e^{q_{1}(n-p)}\right) \mid \\
= & \mid p_{1}(n)-q_{1}(n)-a_{11}(n) \xi_{1}(n)\left(p_{1}(n)-q_{1}(n)\right)-a_{12}(n) \xi_{2}(n)\left(p_{2}(n)-q_{2}(n)\right) \\
& -b_{1}(n) e^{p_{1}(n)} \sum_{p=0}^{M} k_{2}(p)\left[\eta_{2}(n-p)\left(p_{2}(n-p)-q_{2}(n-p)\right)\right] \\
& -b_{1}(n) \xi_{1}(n)\left(p_{1}(n)-q_{1}(n)\right) \sum_{p=0}^{M} k_{2}(p) e^{q_{2}(n-p)} \mid \\
& +\mid p_{2}(n)-q_{2}(n)-a_{21}(n) \xi_{1}(n)\left(p_{1}(n)-q_{1}(n)\right)-a_{22}(n) \xi_{2}(n)\left(p_{2}(n)-q_{2}(n)\right) \\
& -b_{2}(n) e^{p_{2}(n)} \sum_{p=0}^{M} k_{1}(p)\left[\eta_{1}(n-p)\left(p_{1}(n-p)-q_{1}(n-p)\right)\right] \\
& +b_{2}(n) \xi_{2}(n)\left(p_{2}(n)-q_{2}(n)\right) \sum_{p=0}^{M} k_{1}(p) e^{q_{1}(n-p)} \mid \\
\leq & \left\{\left|1-a_{11}(n) \xi_{1}(n)\right|+b_{1}^{u} k_{2}^{u} M_{1} M_{1} M_{2} \sum_{p=0}^{M}\left|p_{2}(n-p)-q_{2}(n-p)\right| .\right. \\
\leq & \left|1-a_{11}(n) \xi_{1}(n)\right| \cdot\left|p_{1}(n)-q_{1}(n)\right|+a_{12}^{u} M_{2}\left|p_{2}(n)-q_{2}(n)\right| \\
& +b_{1}^{u} k_{2}^{u} M_{1} M_{2} M_{2} \sum_{p=0}^{M}\left|p_{2}(n-p)-q_{2}(n-p)\right|+b_{1}^{u} k_{2}^{u} M_{1} M_{2}(M+1)\left|p_{1}(n)-q_{1}(n)\right| \\
& +\left|1-a_{22}(n) \xi_{2}(n)\right| \cdot\left|p_{2}(n)-q_{2}(n)\right|+a_{21}^{u} M_{1}\left|p_{1}(n)-q_{1}(n)\right| \\
& +b_{2}^{u} k_{1}^{u} M_{1} M_{2} \sum_{p=0}^{M}\left|p_{1}(n-p)-q_{1}(n-p)\right|+b_{2}^{u} k_{1}^{u} M_{1} M_{2}(M+1)\left|p_{2}(n)-q_{2}(n)\right| \\
& \left\{1-a_{22}(n) \xi_{2}(n) \mid+b_{2}^{u} k_{1}^{u} M_{1} M_{2}(M+1)+a_{12}^{u} M_{2}\right\} \cdot\left|p_{2}(n)-q_{2}(n)\right| \\
& \\
& \\
&
\end{aligned}
$$

Based on (B.11), we calculate the difference of $V$ along the solution of system (B.3)

$$
\begin{aligned}
\Delta V_{(\mathrm{B} .3)}(n)= & V(n+1)-V(n) \\
= & \left|p_{1}(n+1)-q_{1}(n+1)\right|+\left|p_{2}(n+1)-q_{2}(n+1)\right| \\
& +\sum_{p=0}^{M} \sum_{m=n+1-p}^{n} b_{2}^{u} k_{1}^{u} M_{1} M_{2}\left|p_{1}(m)-q_{1}(m)\right| \\
& +\sum_{p=0}^{M} \sum_{m=n+1-p}^{n} b_{1}^{u} k_{2}^{u} M_{1} M_{2}\left|p_{2}(m)-q_{2}(m)\right|
\end{aligned}
$$




$$
\begin{aligned}
& -\left|p_{1}(n)-q_{1}(n)\right|-\left|p_{2}(n)-q_{2}(n)\right|-\sum_{p=0}^{M} \sum_{m=n-p}^{n-1} b_{2}^{u} k_{1}^{u} M_{1} M_{2}\left|p_{1}(m)-q_{1}(m)\right| \\
& -\sum_{p=0}^{M} \sum_{m=n-p}^{n-1} b_{1}^{u} k_{2}^{u} M_{1} M_{2}\left|p_{2}(m)-q_{2}(m)\right| \\
& \leq\left\{\left|1-a_{11}(n) \xi_{1}(n)\right|+b_{1}^{u} k_{2}^{u} M_{1} M_{2}(M+1)+a_{21}^{u} M_{1}\right\} \cdot\left|p_{1}(n)-q_{1}(n)\right| \\
& +\sum_{p=0}^{M} b_{2}^{u} k_{1}^{u} M_{1} M_{2}\left|p_{1}(n-p)-q_{1}(n-p)\right| \\
& +\left\{\left|1-a_{22}(n) \xi_{2}(n)\right|+b_{2}^{u} k_{1}^{u} M_{1} M_{2}(M+1)+a_{12}^{u} M_{2}\right\} \cdot\left|p_{2}(n)-q_{2}(n)\right| \\
& +\sum_{p=0}^{M} b_{1}^{u} k_{2}^{u} M_{1} M_{2}\left|p_{2}(n-p)-q_{2}(n-p)\right| \\
& +\sum_{p=0}^{M} \sum_{m=n+1-p}^{n} b_{2}^{u} k_{1}^{u} M_{1} M_{2}\left|p_{1}(m)-q_{1}(m)\right| \\
& +\sum_{p=0}^{M} \sum_{m=n+1-p}^{n} b_{1}^{u} k_{2}^{u} M_{1} M_{2}\left|p_{2}(m)-q_{2}(m)\right| \\
& -\left|p_{1}(n)-q_{1}(n)\right|-\left|p_{2}(n)-q_{2}(n)\right|-\sum_{p=0}^{M} \sum_{m=n-p}^{n-1} b_{2}^{u} k_{1}^{u} M_{1} M_{2}\left|p_{1}(m)-q_{1}(m)\right| \\
& -\sum_{p=0}^{M} \sum_{m=n-p}^{n-1} b_{1}^{u} k_{2}^{u} M_{1} M_{2}\left|p_{2}(m)-q_{2}(m)\right| \\
& =\left\{\left|1-a_{11}(n) \xi_{1}(n)\right|+\left(b_{1}^{u} k_{2}^{u}+b_{2}^{u} k_{1}^{u}\right) M_{1} M_{2}(M+1)\right. \\
& \left.+a_{21}^{u} M_{1}-1\right\} \cdot\left|p_{1}(n)-q_{1}(n)\right| \\
& +\left\{\left|1-a_{22}(n) \xi_{2}(n)\right|+\left(b_{1}^{u} k_{2}^{u}+b_{2}^{u} k_{1}^{u}\right) M_{1} M_{2}(M+1)\right. \\
& \left.+a_{12}^{u} M_{2}-1\right\} \cdot\left|p_{2}(n)-q_{2}(n)\right| \\
& \leq-\left\{1-\max \left(\left|1-a_{11}^{l} m_{1}\right|,\left|1-a_{11}^{u} M_{1}\right|\right)-\left(b_{1}^{u} k_{2}^{u}+b_{2}^{u} k_{1}^{u}\right)(M+1) M_{1} M_{2}\right. \\
& \left.-a_{21}^{u} M_{1}\right\} \cdot\left|p_{1}(n)-q_{1}(n)\right| \\
& -\left\{1-\max \left(\left|1-a_{22}^{l} m_{2}\right|,\left|1-a_{22}^{u} M_{2}\right|\right)-\left(b_{1}^{u} k_{2}^{u}+b_{2}^{u} k_{1}^{u}\right)(M+1) M_{1} M_{2}\right. \\
& \left.-a_{12}^{u} M_{2}\right\} \cdot\left|p_{2}(n)-q_{2}(n)\right| \\
& \leq-\theta\left(\left|p_{1}(n)-q_{1}(n)\right|+\left|p_{2}(n)-q_{2}(n)\right|\right) \\
& \leq-\theta \sqrt{\left(p_{1}(n)-q_{1}(n)\right)^{2}+\left(p_{2}(n)-q_{2}(n)\right)^{2}} \\
& =-\theta\left(\left|Y_{n}(0)-Z_{n}(0)\right|\right) \text {, }
\end{aligned}
$$

where $\theta=\min \left\{1-\max \left(\left|1-a_{11}^{l} m_{1}\right|,\left|1-a_{11}^{u} M_{1}\right|\right)-\left(b_{1}^{u} k_{2}^{u}+b_{2}^{u} k_{1}^{u}\right)(M+1) M_{1} M_{2}-a_{21}^{u} M_{1}\right.$, $\left.1-\max \left(\left|1-a_{22}^{l} m_{2}\right|,\left|1-a_{22}^{u} M_{2}\right|\right)-\left(b_{1}^{u} k_{2}^{u}+b_{2}^{u} k_{1}^{u}\right)(M+1) M_{1} M_{2}-a_{12}^{u} M_{2}\right\}$ is a positive constant. Let $c \in C\left(\mathbb{R}^{+}, \mathbb{R}^{+}\right), c(x)=\theta x$, thus condition (3)' in Remark 2.5 is satisfied. Based on Lemma 2.4 and Remark 2.5 , there exists a unique uniformly asymptotically stable almost periodic solution $\left(p_{1}(n), p_{2}(n)\right)$ of system (B.1), that is, there is a unique uniformly 
asymptotically stable positive almost periodic solution $\left(x_{1}^{*}(n), x_{2}^{*}(n)\right)$ of system $(1.1)$ which satisfies $m_{i} \leq x_{i}^{*}(n) \leq M_{i}, i=1,2$, for all $n \in \mathbb{K}$. The proof is complete.

\section{Competing interests}

The authors declare that they have no competing interests.

\section{Authors' contributions}

Each of the authors, QW and ZL, contributed to each part of this work equally and read and approved the final version of the manuscript.

\section{Acknowledgements}

The work is supported by the National Natural Science Foundation of China (Nos. 11261017, 61261044), the Key Project of Chinese Ministry of Education (No. 212111), the Scientific Research Foundation of the Education Department of Hubei Province of China (No. B20111906) and the Key Subject of Hubei Province (Forestry).

Received: 5 July 2013 Accepted: 29 August 2013 Published: 07 Nov 2013

\section{References}

1. Chao, L, Levin, BR: Structured habitats and the evolution of anticompetitor toxins in bacteria. Proc. Natl. Acad. Sci. USA 78, 6324-6328 (1981)

2. Cole, JJ: Interactions between bacteria and algae in aquatic ecosystems. Ann. Rev. Ecolog. Syst. 13, 291-314 (1982)

3. Kitaguchi, H, Hiragushi, N, Mitsutani, A, Yamaguchi, M, Ishida, Y: Isolation of an algicidal marine bacterium with activity against the harmful dinoflagellate Heterocapsa circulatisquama (Dinophyceae). Phycologia 40, 275-279 (2001)

4. Turner, JT, Tester, PA: Toxic marine phytoplankton, zooplankton grazers, and pelagic food webs. Limnol. Oceanogr. 42, 1203-1214 (1997)

5. Folt, C, Goldman, CR: Allelopathy between zooplankton: a mechanism for interference competition. Science 213 1133-1135 (1981)

6. Rice, EL: Allelopathy, 2nd edn. Academic Press, New York (1984)

7. Liu, ZJ, Wu, JH, Chen, YP, Haque, M: Impulsive perturbations in a periodic delay differential equation model of plankton allelopathy. Nonlinear Anal., Real World Appl. 11, $432-445$ (2010)

8. Liu, ZJ, Hui, J, Wu, JH: Permanence and partial extinction in an impulsive delay competitive system with the effect of toxic substances. J. Math. Chem. 46, 1213-1231 (2009)

9. Tian, CR, Lin, ZG: Asymptotic behavior of solutions of a periodic diffusion system of plankton allelopathy. Nonlinear Anal., Real World Appl. 3, 1581-1588 (2010)

10. He, MX, Chen, FD, Li, Z: Almost periodic solution of an impulsive differential equation model of plankton allelopathy. Nonlinear Anal., Real World Appl. 11, 2296-2301 (2010)

11. Chen, YP, Chen, FD, Li, Z: Dynamics behaviors of a general discrete nonautonomous system of plankton allelopathy with delays. Discrete Dyn. Nat. Soc. 2008, Article ID 310425 (2008)

12. $\mathrm{Li}, \mathrm{Z}, \mathrm{Chen}, \mathrm{FD}, \mathrm{He}, \mathrm{MX}$ : Global stability of a delay differential equations model of plankton allelopathy. Appl. Math. Comput. 218, 7155-7163 (2012)

13. Liu, ZJ, Chen, LS: Positive periodic solution of a general discrete non-autonomous difference system of plankton allelopathy with delays. J. Comput. Appl. Math. 197, 446-456 (2006)

14. Liu, ZJ, Chen, LS: Periodic solution of a two-species competitive system with toxicant and birth pulse. Chaos Solitons Fractals 32, 1703-1712 (2007)

15. Qin, WJ, Liu, ZJ: Asymptotic behaviors of a delay difference system of plankton allelopathy. J. Math. Chem. 48, 653-675 (2010)

16. Niu, CY, Chen, XX: Almost periodic sequence solutions of a discrete Lotka-Volterra competitive system with feedback control. Nonlinear Anal., Real World Appl. 10, 3152-3161 (2009)

17. Zhang, TW, Li, YK, Ye, Y: Persistence and almost periodic solutions for a discrete fishing model with feedback control. Commun. Nonlinear Sci. Numer. Simul. 16, 1564-1573 (2011)

18. Wang, Z, Li, YK: Almost periodic solutions of a discrete mutualism model with feedback controls. Discrete Dyn. Nat. Soc. 2010, Article ID 286031 (2010)

19. Li, YK, Zhang, TW: Almost periodic solution for a discrete hematopoiesis model with time delay. Int. J. Biomath. 5 , Article ID 1250003 (2012)

20. Li, Z, Chen, FD, He, MX: Almost periodic solutions of a discrete Lotka-Volterra competition system with delays. Nonlinear Anal., Real World Appl. 12, 2344-2355 (2011)

21. Muroya, Y: Persistence and global stability in discrete models of Lotka-Volterra type. J. Math. Anal. Appl. 330, 24-33 (2007)

22. Zhang, SN, Zheng, G: Almost periodic solutions of delay difference systems. Appl. Math. Comput. 131, 497-516 (2002)

23. Yuan, R, Hong, JL: The existence of almost periodic solutions for a class of differential equations with piecewise constant argument. Nonlinear Anal., Theory Methods Appl. 28, 1439-1450 (1997)

24. He, CY: Almost Periodic Differential Equations. Higher Education Press, Beijing (1992) (Chinese version)

10.1186/1687-1847-2013-283

Cite this article as: Wang and Liu: Uniformly asymptotic stability of almost periodic solutions for a delay difference system of plankton allelopathy. Advances in Difference Equations 2013, 2013:283 